\title{
Perceptions et Motivation Des Élèves Et Des Enseignants Sur L'enseignement-Apprentissage Du Français Au Rwanda
}

Léonce NSABUMUREMYI* $^{1}$, Froduald NDAYAMBAJE ${ }^{1}$ and Aimable RUGIGANA ${ }^{1}$ ${ }^{1}$ University of Rwanda-College of Education, Rwanda

\section{Article History}

Received: 20.08.2020

Accepted: 06.09.2020

Published: 30.09 .2020

Journal homepage:

https://www.easpublisher.com/easjhcs

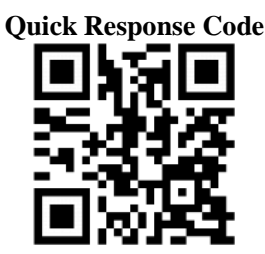

Abstract: Cette étude s'est proposée de contribuer à la compréhension de la situation actuelle du français dans l'enseignement secondaire et de proposer quelques voies d'issues aux problèmes que connaissent l'enseignement et l'apprentissage de cette langue. Pour arriver à ce but, la méthode mixte a été employée. Pour ce faire un questionnaire autodirigé a été administré à 180 élèves des écoles secondaires du centre-ville du District de Nyanza et des entretiens individuels ont été menés auprès de 6 enseignants des mêmes écoles. Les résultats ont montré que les conditions d'apprentissage sont positifs pour les aspects relatifs à la perception de l'utilité du français, appui matériel des parents et appréciation des méthodes d'enseignement; mais négatifs pour les aspects relatifs aux soutiens des parents dans les devoirs, l'insuffisance d'encouragement des pairs et de l'entourage quant à l'utilisation du français. Quant à la motivation à apprendre le français, les résultats montrent que les élèves sont en général motivés à apprendre le français malgré quelques aspects démotivants comme la perception du français comme langue difficile, peu d'encouragement à pratiquer la langue et difficiles conditions d'apprentissage comme le manque du matériel approprié. Eu égards aux résultats, quelques stratégies d'amélioration des conditions d'enseignement-apprentissage du français ont été formulées comme entre autres l'amélioration du statut de cette langue et un travail de changement d'attitudes à son égard.

Keywords: perception, motivation, apprentissage, enseignement, FLE.

Copyright $\odot 2020$ The Author(s): This is an open-access article distributed under the terms of the Creative Commons Attribution 4.0 International License (CC BY-NC 4.0) which permits unrestricted use, distribution, and reproduction in any medium for non-commercial use provided the original author and source are credited.

\section{INTRODUCTION}

Le français a été introduit au Rwanda par les colonisateurs belges depuis à peu près un siècle pour servir d'outil de communication dans l'administration coloniale et dans l'enseignement. Depuis les années 1916 le français fut enseigné à la fois comme matière et comme langue d'instruction jusqu'en 2010. Depuis lors, la langue d'enseignement est désormais l'anglais. Le français est resté à son statut de matière enseignée dans la combinaison FEK (Français- Anglais-Kinyarwanda). Il était aussi cours facultatif au Tronc commun de l'enseignement secondaire.

Cependant, le nouveau programme d'enseignement de 2015 basé sur les compétences (Competence Based Curriculum) prévoit le retour du français dans l'enseignement au second cycle du Primaire, dans le Tronc Commun du secondaire comme cours obligatoire et dans le second cycle du secondaire comme cours obligatoire dans les combinaisons où le français est l'une des composantes et facultatif dans d'autres combinaisons (REB, 2015). Maintenant que le français revient sur la scène scolaire, on pourrait se demander comment les élèves et les enseignants perçoivent ce cours, son apprentissage et son enseignement et comment sont-ils motivés à enseigner et à apprendre cette langue.

Les recherches en didactique des langues sont généralement d'accord sur le fait que la motivation est l'un des plus importants (Karolina, 2007). Dans un contexte scolaire, la motivation est définie comme un état dynamique qui a ses origines dans les perceptions qu'un élève a de lui-même et de son environnement et qui l'incite à choisir une activité, à s'y engager et à persévérer dans son accomplissement afin d'atteindre un but (Pintrich et Schrauben, 1992).

D’après Deci et Ryan (1985), il existe deux types de motivations : la motivation intrinsèque et la motivation extrinsèque. La motivation intrinsèque réfère à une pratique volontaire d'une activité pour l'intérêt qu'elle présente en elle-même et sans intervention de récompense extérieure. Cette motivation implique le plaisir, le contentement, et la satisfaction. La motivation extrinsèque, quant à elle, réfère à un engagement dans un but non inhérent à l'activité, soit en vue de retirer quelque chose déplaisant, soit pour éviter quelque chose de déplaisant. La motivation extrinsèque $\mathrm{a}$, par essence, une fonction instrumentale. 
Partant de cette définition, la motivation s'avère d'une importance capitale dans tout processus d'enseignement-apprentissage et tout spécialement dans celui des langues. Elle constitue l'énergie qui permet de s'engager et de persévérer dans l'effort à long terme qu'exige l'apprentissage d'une langue.

La motivation est un phénomène très complexe. D'après Gadner (2010), la motivation est un agrégat de trois variables que sont le désir d'apprendre la langue, les attitudes envers l'apprentissage de la langue et l'intensité motivationnelle c'est-à-dire le degré d'effort investi dans cet apprentissage. Ainsi, un niveau élevé de motivation stimule l'apprentissage, et la réussite dans l'apprentissage de la langue étrangère aide l'apprenant à maintenir une motivation déjà existante. Au contraire, si la motivation est basse, les résultats deviennent mauvais et la motivation sera encore plus basse (Ellis, 1994).

Il est à signaler que la motivation de l'élève n'est pas dissociable de celle de l'enseignant. Selon Prot(1997), la motivation des élèves et celle des enseignants sont liées. Même si la motivation est un élément essentiel dans l'enseignement-apprentissage d'une langue étrangère, elle n'est pas le seul critère pour la réussite. D'autres éléments comme les connaissances antérieures de l'apprenant, sa personnalité, son âge, sa capacité et ses attitudes sont également à tenir en considération (Benoît, 2006). Ces éléments sont souvent regroupés sous le terme de perceptions.

De plus, la façon dont l'élève perçoit l'enseignement peut aussi affecter sa motivation (Ryan, \& Grolnick, 1986). Certains auteurs ont étudié cette question en faisant l'analyse des perceptions et de leur rôle dans la motivation scolaire. Par exemple Viau (2009) propose d'étudier la motivation des élèves dans l'apprentissage en relation avec leurs perceptions.

Les concepts de perception et de motivation sont donc intimement liés (Gagné et Popica 2017) qu'il n'est pas aisé de parler de l'un en mettant de côté l'autre. En effet, la façon dont l'élève perçoit l'enseignement peut affecter sa motivation (Ryan \& Grolnick, 1986) et le degré de motivation influence la perception qu'un élève a de son apprentissage.

Dans cette étude, le terme perception est employé pour désigner le processus par lequel les élèves et les enseignants acquièrent de l'information sur diverses expériences en classe de FLE : les enseignants envers les élèves et vice versa, les enseignants et les élèves envers la discipline de FLE, et envers leurs propres compétences.

Plusieurs chercheurs et auteurs ont parlé de la langue française au Rwanda. On pourrait notamment indiquer Gafaranga (1984) qui s'est penché sur la naissance du Kinyafrancais, un parler né du mixage du kinyarwanda et du francais surtout chez l'élite intellectuel rwandais. Kabanza (2004) a quant à lui etudié comment le bilguisme pourait être redynamisé en tenant compte de la cohabitation du francais et de l'Angalais comme langues étrangères au Rwanda. D'autre part, Rurangirwa (2010) et Munyankesha (2011) se sont interessés aux politiques linguistiques au Rwanda ainsi qu'à l'avenir du français dans le contexte de ces politiques. On peut également mentionner Yanzigiye et Niyomugabo (2013) qui ont analysé la pratique sociale du français au Rwanda. Enfin, il y a Ntakirutimana (2014) qui lui s'est intéressé à l'analyse de la dynamique des langues dont le français dans l'enseignement supérieur rwandais.

Cependant, la plupart de ces travaux sur le français sont surtout du domaine de la sociolinguistique et de l'aménagement linguistique. Force est de constater que les publications qui abordent le français au Rwanda du point de vue didactique sont quasi inexistants.

Ainsi cette étude s'est proposée de trouver le français dans son milieu d'enseignement-apprentissage pour analyser comment les enseignants et les élèves le perçoivent et étudier leur motivation envers cette langue, compte tenu de son retour sur la scène scolaire. Elle s'est attelée à contribuer à la compréhension de la situation actuelle du français dans l'enseignement secondaire et de proposer quelques voies d'issues aux problèmes que connaissent l'enseignement et l'apprentissage de cette langue.

\section{Methodologie}

Cette étude sur la perception et la motivation des élèves et des enseignants sur l'enseignement du français au Rwanda a été menée dans les écoles secondaires localisées dans le centre-ville du district de Nyanza de la province du Sud. Le choix du centre-ville de Nyanza a été motivé surtout par son emplacement géographique. En effet, c'est un milieu semi urbain accessible, dont le nombre d'établissements permet une collecte de données diversifiées. Ainsi cette étude a porté sur trois établissements secondaires à savoir : Collège du Christ-Roi (une école publique d'excellence), École Secondaire Saint Peter Igihozo (une école privée), Groupe Scolaire Nyanza B (une école publique d'éducation de base de neuf ans).

Il n'a nullement été question de généraliser les résultats au reste du pays mais plutôt d'apporter une contribution à la compréhension de la situation de l'enseignement-apprentissage du français au Rwanda. Comme le disent Quivy \& Campenhoudt ( 1995), il est possible d'obtenir des informations fiables sur une grande population en se servant d'une partie représentative de cette population. Ainsi, notre échantillon est composé de 180 élèves et de 6 
enseignants de français de ces établissements scolaires ci-haut mentionnés.

Pour la collecte des données, la méthode mixte a été adoptée. Il y a eu, d'une part, des entretiens individuels avec six enseignants de français. Ces entretiens ont été choisis parce que, comme le souligne Patton (2002), ils aident à accéder aux pensées de quelqu'un et à l'ampleur de ces dernières. Ce qui cadre bien avec l'objectif de cette étude. D'autre part, il y a eu un questionnaire distribué à 180 élèves qui ont été choisis par échantillonnage par quotas pour participer à l'étude. Le questionnaire autodirigé a été préféré en raison du nombre de participants et de la nature du sujet de recherche qui exigeait des données quantitatives et qualitatives (Kothari, 2004).

Pour l'analyse des résultats, comme l'étude s'inscrit dans une démarche mixte (qualitative et quantitative), l'analyse thématique de contenu a été utilisée pour l'analyse des données de différents entretiens avec les enseignants et des questions ouvertes $\mathrm{du}$ questionnaire des élèves. Pour des données quantitatives, le logiciel SPSS nous a permis d'obtenir des statistiques descriptives comme l'analyse des fréquences et des pourcentages.

Il faut aussi signaler que cette étude s'est conformée à la réglementation en vigueur concernant le code éthique en matière de recherche et que les chercheurs ont obtenu l'autorisation de mener l'étude des instances de l'Université du Rwanda.

\section{Présentation et discussion des résultats}

Cette section focalise sur les deux thèmes principaux, à savoir: perceptions des élèves et des enseignants à l'égard de l'enseignement -apprentissage du FLE et motivation des élèves et des enseignants pour l'enseignement et l'apprentissage du FLE.

Les résultats présentés proviennent d'un échantillon de 180 élèves dont 77 garçons (42.8\%) et 103 filles $(57.2 \%)$ et de 6 enseignants dont 4 hommes et 2 femmes. La majorité des élèves qui ont répondu au questionnaire étudient au niveau inférieur (communément appelé Tronc Commun) soit 118 sur $180(65.56 \%)$. L'âge des élèves qui ont participé à cette étude s'étend de 12 à plus de 18 ans avec le plus grand nombre âgé de 15 ans (25\%). Nos répondants ont dit avoir commencé l'apprentissage du français à partir de différentes classes, de la maternelle en $6^{\text {ème }}$ année primaire, avec seulement $15 \%$ qui ont commencé à l'apprendre à partir du secondaire.

\section{Perceptions des élèves et des enseignants du secondaire à l'égard de l'enseignement et l'apprentissage du français.}

Dans l'objectif d'analyser les perceptions des élèves et des enseignants du secondaire à l'égard de l'enseignement et de l'apprentissage du français, nous avons voulu savoir comment les élèves et les enseignants perçoivent la langue française et les conditions de son enseignement-apprentissage dans le contexte rwandais.

\section{Perception des élèves et des enseignants vis-à-vis du français}

En premier lieu, il a été question de savoir ce que les élèves et les enseignants du secondaire pensent de l'utilité du français dans leur vie présente et future. Les résultats montrent qu'en général la majorité des élèves qui ont répondu au questionnaire reconnaissent l'utilité de la langue française (87.2\%). Ceci fait penser qu'ils sont généralement motivés à apprendre cette langue. Le même résultat se fait remarquer dans l'interview avec les enseignants. Comme l'a déclaré l'un des enseignants interviewés: «le français revêt d'une importance capitale dans la mesure où c'est une langue internationale qui, en plus de cela, est très parlée dans la région d'Afrique centrale où se situe le Rwanda ». La perception tant des enseignants que des élèves quant à l'utilité du français langue seconde s'alignent avec ce que diverses recherches ont prouvé, à savoir que l'apprentissage d'une deuxième langue présente des avantages considérables tant sur le plan cognitif que sur le plan des résultats scolaires (Adam, 2005; Genesee, 2008; Lapkin, Mady et Arnott, 2009 ; Netten et Germain, 2005).

Cependant, la proportion des élèves qui ne voient pas l'utilité de cette langue (12.8\%) n'est pas du tout négligeable pour un pays membres de la famille francophone. Cela résulterait du fait que depuis 2009 le français ne jouit plus du statut de langue d'enseignement et qu'il était même relégué au second plan jusqu'à l'introduction du nouveau curriculum basé sur les compétences, en 2015 (Munyankesha, 2011). Ceci implique que les enseignants ainsi que leurs partenaires dans l'enseignement-apprentissage du français devraient faire un effort pour changer les croyances des élèves en les intéressant davantage à l'utilité du français.

Deuxièmement, nous avons voulu savoir ce que pensent les élèves et les enseignants de leur utilisation du français en dehors de la classe. Les données montrent que seulement $50 \%$ des élèves admettent qu'il leur arrive d'utiliser le français en dehors de la classe. Les enseignants eux aussi affirment que non seulement les élèves n'utilisent pas le français à l'école mais qu'eux-mêmes l'utilisent très rarement à l'école en dehors de la classe. Ceci montre que même si les élèves et les enseignants reconnaissent l'utilité potentielle de cette langue, ils ne trouvent que peu d'occasion de l'utiliser en pratique. La raison serait que les Rwandais communiquent aisément en Kinyarwanda, langue maternelle et nationale utilisée par tous (Munyankesha, 2011). 
Cette insuffisance de l'utilisation pratique du français par les enseignants, les élèves ainsi que par les rwandais en général pose un sérieux problème à son apprentissage efficace. En effet, comme l'affirment diverses recherches, l'apprentissage efficace d'une langue seconde nécessite la disponibilité des occasions d'interactions authentiques avec des personnes qui la parlent ainsi que des opportunités d'action où les compétences linguistiques peuvent se développer (MacFarlane , 2001 ; Scallon, 2004).

\section{Perception des conditions d'enseignement et d'apprentissage du FLE}

Dans le souci de cerner les perceptions des élèves et des enseignants quant aux conditions d'apprentissage du français, nous avons voulu examiner si les élèves et les enseignants considèrent les conditions d'enseignement-apprentissage favorables, donc motivant, à apprendre le français.

Premièrement, concernant la satisfaction des élèves à l'égard des méthodes que les professeurs utilisent pour enseigner le français, les données montrent que $64.4 \%$ des participants à notre recherche se disent satisfaits. Ceci parait intéressant bien que la proportion de ceux qui se disent insatisfaits est, elle aussi, non moins importante, $35.6 \%$. Ceci rejoint ce que les enseignants ont déclaré concernant leur capacité à faciliter les élèves dans leur apprentissage du français où la majorité des enseignants interviewés affirment disposer suffisamment de compétences à enseigner le français. Il y a donc lieu de penser que les méthodes d'enseignement sont quand même relativement encourageantes. D'ailleurs, l'enseignant joue un rôle important dans la motivation à apprendre une langue étrangère, particulièrement par les activités qu'il choisit, la dynamique de la classe qu'il crée et l'attitude envers la classe qu'il inspire à travers ses méthodes d'enseignement (Arnold, 2006; Viau, 2009).

Deuxièmement, à la question de savoir comment sont perçus par leurs collègues les élèves qui essaient de faire usage du français dans leur communication pendant la récréation, les données montrent que seulement $45.56 \%$ des répondants trouvent que les élèves apprécient leurs collègues qui utilisent le français hors de la situation de classe, dans la récréation. La majorité de nos répondants, $54.44 \%$, ont dit qu'ils n'apprécient pas leurs collègues qui utilisent le français en dehors de la classe. Ceci montre que les élèves eux même constituent pour une part non négligeable un environnent décourageant pour l'apprentissage du français. En effet, la désapprobation sociale peut être un facteur inhibant dans l'apprentissage d'un comportement, ici linguistique. Ce résultat est inquiétant pour la motivation, et par conséquent, le succès dans l'apprentissage du français. En effet, il semble que les élèves ne sont pas encouragés à pratiquer des interactions dans cette langue, ce qui serait par ailleurs l'une des conditions pour l'efficacité de son apprentissage (Lightbown et Spada, 2006 ; Mady et Arnott, 2010).

Du côté des enseignants, l'usage du français en dehors de la situation de classe est plutôt encourageant car comme le dit cet enseignant interviewé «les collègues se montrent intéressés et souvent même exaltés quand ils entendent un collègue s'exprimer en français ». Mais certains des enseignants interrogés disent que les vieux enseignants, avec un antécédent francophone, se montrent plus intéressés que les jeunes sans cet antécédent.

A part l'auto-encouragement entre les collègues, nous nous sommes aussi intéressés à l'encouragement par les parents des élèves et la communauté en général. Concernant la question de savoir si les parents encouragent les élèves à apprendre le français, les données montrent que $82.3 \%$ des élèves estiment que leurs parents les encouragent à apprendre le français contre $17.7 \%$ qui estiment délaissés par leurs parents dans leur processus d'apprentissage du français. Quant à l'appui matériel dont bénéficient les élèves de la part de leurs parents, les données montrent qu'une légère majorité $(67.78 \%)$ estime que leurs parents leur donnent le matériel suffisant pour l'apprentissage du français.

Ce résultat peut être considéré comme une bonne nouvelle car comme le dit le Ministère de l'Education de l'Ontario (2013), l'apprentissage du FLE n'est efficace que quand il y a implication des parents, de divers intervenants dans l'éducation et de la communauté en générale. En effet, les parents jouent un rôle déterminant en façonnant les attitudes et les valeurs de leurs enfants, ils aident ces derniers à se fixer des objectifs et à se tourner vers l'avenir.

Les enseignants, quant à eux, voient que l'appui dont bénéficient les enseignants et les élèves pour l'enseignement et l'apprentissage du français n'est pas suffisant. La plupart des enseignants interviewés ont dit que les élèves ne sont pas suffisamment appuyés, ni par leurs parents ni par leurs écoles. Ils ont pointé du doigt l'insuffisance du matériel scolaire, des manuels scolaires ainsi des moyens audio-visuels. Surtout, il a été souligné que les manuels scolaires utilisés ne sont pas adaptés au nouveau curriculum et qu'il y a un manque notoire de documents pour la lecture tels que livres, romans et journaux.

Troisièmement, s'agissant de l'appui des parents aux élèves, en ce qui concerne les devoirs, les données montrent que seulement $58.3 \%$ des élèves affirment recevoir de l'aide des parents pour les révisions et les devoirs, alors que $41.7 \%$ disent ne recevoir aucune aide. Force est de constater qu'il existe 
peu d'encouragement de la part des parents quant à l'apprentissage du français surtout en ce qui concerne les devoirs à domicile. Certains des enseignants interviewés confirment cette information: "Quand nous leurs demandons pourquoi ils n'ont pas fait le devoir de français, certains répondent qu'il n'y a personne à la maison pour les aider, les autres disent que personne ne veut les aider sous prétexte que le français est très difficile », nous a déclaré un des enseignants. Cette insuffisance de soutien des parents en ce qui concerne les devoirs constitue un obstacle à l'apprentissage du FLE d'autant plus que comme le souligne le Ministère de l'Education de l'Ontario (2013), il est important que les parents soutiennent les élèves tout au long de leur processus d'apprentissage, y compris leur faciliter à faire leurs devoirs et cela ne nécessite pas une commande de la langue mais plutôt une attitude positive et de l'intérêt.

Il a été aussi question de savoir si les élèves sentent que la communauté soutient l'apprentissage du français par son appréciation de l'usage du français. Les données montrent que $66.2 \%$ des élèves trouvent que l'entourage (la communauté) apprécie l'usage du français. Ceci montre que l'environnement des élèves n'est pas suffisamment encourageant. Les enseignants interviewés sont du même avis que l'environnement n'est pas suffisamment encourageant quant à l'enseignement et l'apprentissage du français.

Enfin, il a été question de savoir ce que les élèves pensent de la disponibilité des outils de Technologie de l'information et de la communication éducative (TICE) pour l'apprentissage du français dans leurs écoles. Les données montrent que seulement 7.8\% des élèves estiment que leurs écoles mettent à leur disposition des outils nécessaires pour l'apprentissage $\mathrm{du}$ français. Les enseignants interrogés le confirment aussi, comme nous l'a déclaré l'un d'eux: «Il y a un manque criant d'outils de Technologie de l'information et de communication ». Cet état de chose pourrait constituer un facteur démotivant quant à l'apprentissage du français d'où il est souhaitable que les instances habiletés fassent un effort dans ce domaine.

\section{Etat de la motivation des élèves et des enseignants du secondaire quant à l'enseignement et l'apprentissage du français.}

Tout d'abord, à la question de savoir comment les élèves perçoivent la facilité ou la difficulté à apprendre le français, seule la moitié des élèves (50\%) affirment que le français est une langue facile à apprendre. Ceci rejoint ce que les enseignants interviewés affirment que seulement peu d'élèves semblent apprendre le français avec une relative facilité.

De plus, interrogés sur leur appréciation du niveau des élèves en français, tous les enseignants affirment que peu d'élèves ont un niveau souhaitable du français. L'un des enseignants interviewés le dit dans ces termes: «La majorité des élèves ont un niveau de français très bas surtout qu'ils ont appris le français un peu tard, à partir de la première année secondaire».

On en déduit que le français est perçu par la plupart des élèves comme une langue difficile à apprendre, ce qui pourraient affecter négativement leur motivation à l'apprendre. En effet, comme le dit Arnold ( 2006) cité par Longpré ( 2015), l'attitude des apprenants par rapport à la langue qu'ils apprennent a une grande influence sur le succès de cet apprentissage.

Toutefois, à la question de savoir comment les élèves jugent la difficulté du cours de français par rapport aux autres cours, il apparait que même si il est perçu comme une langue relativement difficile, son apprentissage n'est pas le plus difficile des cours du secondaire. En effet, seulement $33.3 \%$ des élèves affirment que le cours de français est le plus difficile des cours du secondaire. Ceci pousserait à penser que les élèves du secondaire, tout en considérant le français comme une langue plus ou moins difficile à apprendre, ils l'estiment plus ou moins abordable.

D'autre part, la majorité des élèves ne soutiennent pas la suppression de ce cours. En effet, seulement $26.1 \%$ contre $73.9 \%$ des répondants affirment qu'ils seraient contents si le cours de français était supprimé. Cela montre que la majorité des élèves $\mathrm{du}$ secondaire aiment apprendre le français. Ainsi, on peut en déduire qu'ils sont en quelque sorte motivés.

Ensuite, à la question de savoir ce que pensent les élèves de leur satisfaction quant aux résultats qu'ils obtiennent dans le cours de français, les données montrent que $60.0 \%$ des répondants sont satisfaits de leurs résultats dans le cours de français. Bien que $40.0 \%$ n'en soient pas satisfaits, il est clair que la motivation par les résultats obtenus en classe reste une possibilité. Ce résultat semble encourageant dans la mesure où comme le souligne Tardieu (2009) et Nzuki Kyuma (2014), les résultats obtenus dans l'apprentissage d'une langue étrangère comptent beaucoup pour la motivation de l'apprenant. Par contre, même si les élèves en général se disent satisfaits de leurs résultats dans le cours de français, les enseignants se montrent inquiets quant aux résultats attendus de leurs élèves à l'examen national de fin d'études du premier cycle du secondaire prévu dans un avenir proche. Par exemple l'un de ceux qui ont été interviewés se dit curieux de voir comment ce cours sera évalué à l'examen national en ces termes: « Peutêtre qu'ils (les évaluateurs) tiendront compte des conditions d'apprentissage! Sinon, s'ils tiennent absolument à évaluer les compétences telles que décrites dans le curriculum, très peu pourront s'en sortir». 
Toujours, dans le souci de connaître la motivation des élèves à apprendre le français, nous avons voulu savoir dans quelle mesure les élèves se sentent capables d'exprimer leurs idées en français, ce qui serait aussi une motivation pour apprendre cette langue. Les données collectées montrent que seulement $43.9 \%$ des élèves se disent capables d'exprimer leurs idées en français. Ce n'est pas étonnant qu'une grande proportion, $56.1 \%$, se sentent incapables de s'exprimer en français dans la mesure où, d'une part, ils sont en cours d'apprentissage et, d'autre part, le français a été suffisamment abandonné et négligé dans l'enseignement secondaire au Rwanda depuis 2009 (Munyankesha, 2011).

Une autre question était de savoir si les élèves se sentent capable d'apprendre le français, ce qui serait aussi une très grande source de motivation. Les données analysées prouvent que $68.3 \%$ des élèves se croient capables d'apprendre le français. Ceci nous démontre leur degré d'appréciation personnelle face à la motivation d'apprendre le français. Cette position est corroborée par les enseignants interviewés dont la plupart ont affirmé que leurs élèves se montrent enthousiastes à apprendre le français bien que dans certains cas cette motivation semble s'effriter suite aux conditions d'apprentissage quelque peu motivant comme le peu de considération dont jouit cette langue.

\section{CONCLUSION}

\section{RECOMMANDATIONS}

Cette étude s'était proposée d'analyser les perceptions des élèves et des enseignants du secondaire à l'égard de l'enseignement et l'apprentissage du français ainsi que de décrire l'état de la motivation des élèves et des enseignants du secondaire quant à l'enseignement et l'apprentissage du français.

Les résultats ont montré que les élèves et les enseignants perçoivent l'enseignement et l'apprentissage du français de façon positive pour certains aspects et négative pour d'autres. Parmi les aspects que les élèves et les enseignants perçoivent comme positifs, on peut citer les méthodes d'enseignement, l'encouragement des parents et leur soutien matériel ainsi que l'approbation sociale de la communauté locale quant à l'apprentissage du français. Par contre, le manque de soutien dans les devoir, l'insuffisante de la provision du matériel scolaire (comme les livres, l'équipement audio-visuel et informatique), la communauté scolaire qui semble désapprouver l'usage du français ainsi que le statut quelque peu considéré du français constituent les aspects négatifs des conditions d'enseignement et d'apprentissage du français.

Aussi, il a été question de décrire l'état de la motivation des élèves et des enseignants du secondaire quant à l'enseignement et l'apprentissage du français. Les résultats ont montré qu'il y a aussi des aspects positifs et négatifs quant à la motivation à enseigner et à apprendre le français. Parmi les aspects positifs, l'étude montre que la majorité d'élèves soutiennent le maintien $\mathrm{du}$ français dans leur curriculum de formation, se disent satisfaits des résultats qu'ils obtiennent dans le cours de français et restent optimistes quant à leur capacité à apprendre la langue française. Quant au côté négatif, l'étude a montré que les élèves perçoivent le français comme une langue difficile à apprendre et les enseignants estiment que le niveau du français est en général très bas pour la plupart des élèves.

En définitive, les résultats de cette étude ont permis
de proposer des stratégies d'amélioration de
l'enseignement-apprentissage du français de façon à
motiver les élèves et les enseignants comme entre
autres:
- Le Gouvernement devrait faire tout son possible pour relever le statut du français en lui accordant une place importante dans le curriculum et en fournissant aux écoles du matériel suffisant et adéquat pour l'apprentissage du français, surtout du matériel audio-visuel et des outils de Technologies d'Information et de Communication en Education (TICE).

- Les élèves, les enseignants, les autorités scolaires et les parents devraient changer d'attitudes à l'égard $\mathrm{du}$ français et contribuer, dans la mesure du possible, à son apprentissage efficace.

Cette étude ne peut pas prétendre avoir épuisé tout ce qui est de la perception et de la motivation des élèves et des enseignants du secondaire vis-à-vis du français au Rwanda. D'autres études pourraient approfondir ce domaine en prenant un plus large échantillon, ou en étendant l'étude au niveau national ou en abordant d'autres aspects influençant la motivation dans l'enseignement-apprentissage des langues étrangères tels que l'âge, le milieu social et les nouvelles technologies de l'information et de la communication.

\section{REFERENCES}

1. Adam, D. (2005). Rapport annuel du Commissariat aux langues officielles. Ottawa- Canada: Travaux publics et services gouvernementaux.

2. Arnold, J. (2006). Comment les facteurs affectifs influencent-ils l'apprentissage d'une langue étrangère? Études de linguistique appliquée, 144(4), 407-425.

3. Arnold, J. (2006). Comment les facteurs affectifs influencent-ils l'apprentissage d'une langue étrangère? Études de linguistique appliquée, 144(4), 407-425.

4. Benoît, G. (2006). La motivation en situation d'apprentissage: les apports de la psychologie de l'éducation. Revue française de pédagogie. 
5. Deci, E. L., \& Ryan R. M. (1985). Intrinsic motivation and self-regulation in human behavior. New York: Plenum Press.

6. Ellis, R. (1994). The Study of Second Language Acquisition. Oxford: Oxford University Press.

7. Gadner, R. (2010). The Social-Educational Model of Second Language Acquisition : A Research Paradigm . Eurosla Yearbook, 237-260.

8. Gafaranga, J. (1984). Le Kinyafrançais, fils légitime et unique du kinyarwanda et du français. Etudes Rwandaises, vol.2, Ruhengeri : UNR.

9. Gagné, P. et Popica, M. (2017). Perceptions et motivation à l'égard du français langue seconde enseigné au Québec. Montréal : Vanier Press.

10. Genesee, F. (2008). Dual language in the global village. In T. W. (éd.), Pathways to multilingualism: Evolving perspectives on immersion education, chapitre 2. Bristol, R.-U: Multilingual Matters.

11. Kabanza, F. (2004). Redynamisation $d u$ bilinguisme précoce au Rwanda. Rapport de DEA. Rouen.

12. Karolina, A. (2007). L'importance de la motivation pour apprendre une langue étrangère - la langue française dans un contexte scolaire. Mémoire en ligne. Växjö Universitet.

13. Kothari, C. (2004). Research Methodology, Methods and Techniques. New Delhi: New Afe International (P) Ltd. Publishers.

14. Lapkin, S., Mady, C., \& Arnott, S. (2009). Research perspectives on core French: A literature review. Canadian Journal of Applied Linguistics, 12(2).

15. Lightbown, P. M., \& Spada, N. (2006). How languages are learned. $3 e$ édition. Oxford: Oxford University Press.

16. Longpré, T. (2015). Les facteurs affectifs dans l'apprentissage du français chez les immigrants allophones adultes à Montréal. Revue canadienne des jeunes chercheures et chercheurs en éducation, 6(2),62-72.

17. MacFarlane, A. (2001). Are brief contact experiences and classroom language learning complementary? . Canadian Modern Language Review, 58, 63-83.

18. Mady, C., \& Arnott, S. (2010). Exploring the "situation" of situational willingness to communicate . A voluntary youth exchange perspective. Canadian Journal of Applied Linguistics ,13(2), 1-25.

19. Ministère de l'Education de l'Ontario (2013). Cadre stratégique pour l'apprentissage du français langue seconde dans les écoles de l'Ontario : de la Maternelle à la $12^{\mathrm{e}}$ année. Ontario, Canada: Ministère de l'Education.

20. Munyankesha, P. (2011). Quel avenir pour le français dans la nouvelle politique linguistique du
Rwanda ? Les Cahiers du GRELCEF. No 2. La Textualisation des langues dans les écritures francophones.

21. Munyankesha, P. (2011). Quel avenir pour le français dans la nouvelle politique linguistique du Rwanda ? Les Cahiers du GRELCEF. No 2. La Textualisation des langues dans les écritures francophones.

22. Netten, J., \& Germain, C. (2005). Pedagogy and second language learning: Lessons learned from Intensive French. Canadian Journal of Applied Linguistics, 8(2), 183-210.

23. Ntakirutimana, E. (2014). La dynamique des langues dans l'enseignement supérieur au Rwanda. Des nouveaux enjeux, une nouvelle dynamique. Synergies Afrique des Grands Lacs no 3, 155-163.

24. Nzuki Kyuma, B. (2014). Les causes de l'abandon de l'étude du français en form deux au Kenya. Journal of Language, Technology \& Entrepreneurship in Africa, 5(1), 42-56.

25. Patton, M. (2002). Quantitative research and Evaluation Methods, 3rd Edition. Sage Publications, Inc.

26. Pintrich, P. R., \& Schrauben, B. (1992). Student's motivational beliefs and their cognitive engagement in classroom academic task. D. $H$. Schunk, et J.L. Meece (dir.), Student Perceptions in the Classroom, Hillsdale: Lawrence Erlbaum, 140183.

27. Prot, B. (1997). Profession motivatrice : réveiller le désir d'apprendre au collège et au lycée. Paris: Ed. Noêsis.

28. Quivy, R., \& Campenhoudt, L. V. (1995). Manuel de recherche en sciences sociales. Paris: Dunod.

29. REB. ( 2015). Programme de français au tronc commun. Kigali.

30. Rurangirwa, S. (2010). Les politiques linguistiques du Rwanda. Enjeu, bilan et perspectives. Thèse de Doctorat en Sciences du langage. Paris : Université de Paris 3.

31. Ryan, R. M., \& Grolnick, W. S. (1986). Origins and pawns in the classroom: Self-report and projective assessments of individual differences in children's perceptions. Journal of Personality and Social Psychology,50, 550-558.

32. Scallon, G. (2004). L'évaluation des apprentissages dans une approche par compétences. Louvain-la-Neuve: De Boeck.

33. Tardieu, C. (2009). « Corriger ou évaluer? Correction or assessment?». La correction dans l'enseignement des langues de spécialité ». Cahiers de l'APLIUT Vol. XXVIII $N^{\circ} 3,16$.

34. Viau, R. (2009). La motivation en contexte scolaire. Bruxelles: De Boeck

35. Yanzigiye, B. \&Niyomugabo, C. (2013). Pratique sociale du français au Rwanda. Synergies Afrique des Grands Lacs, 2, 189-197. 